\title{
Precision control of flow rate in microfluidic channels using photoresponsive soft polymer actuators
}

\author{
Colm Delaney \\ Dublin City University \\ Peter McCluskey \\ Dublin City University \\ Simon Coleman \\ Dublin City University
}

See next page for additional authors

Follow this and additional works at: https://arrow.tudublin.ie/engschmanart

Part of the Mechanical Engineering Commons

\section{Recommended Citation}

Delaney, Colm \& McCluskey, Peter \& Coleman, Simon \& Whyte, Jeffrey \& Kent, Nigel \& Diamond, Dermot. (2017). Precision Control of Flow Rate in Microfluidic Channels Using Photoresponsive Soft Polymer Actuators. Lab Chip. 17. -. 10.1039/C7LC00368D.

This Article is brought to you for free and open access by the School of Manufacturing and Design Engineering at ARROW@TU Dublin. It has been accepted for inclusion in Articles by an authorized administrator of ARROW@TU Dublin. For more information, please contact arrow.admin@tudublin.ie, aisling.coyne@tudublin.ie, gerard.connolly@tudublin.ie.

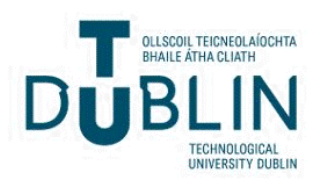




\section{Authors}

Colm Delaney, Peter McCluskey, Simon Coleman, Jeffrey Whyte, Nigel Kent, and Dermot Diamond 


\title{
Lab on a Chip
}

Check for updates

Cite this: Lab Chip, 2017, 17, 2013

\section{Precision control of flow rate in microfluidic channels using photoresponsive soft polymer actuators $\dagger$}

\author{
Colm Delaney, (iD a Peter McCluskey, ${ }^{a}$ Simon Coleman, ${ }^{a}$ Jeffrey Whyte, ${ }^{b}$ \\ Nigel Kent ${ }^{\mathrm{ab}}$ and Dermot Diamond (iD)*a
}

\begin{abstract}
A novel approach that allows control of flow in microfluidic channels with unsurpassed performance using light is described. Valve structures have been created using photoresponsive hydrogels based on spiropyran-functionalised pNIPAAm hydrogels photopolymerised around pillar structures within the channels. Valve actuation is controlled from outside the fluidic system using externally located LEDs. Highly precise and accurate flow rates can be selected by passing real-time flow rate measurements into a PID algorithm. The optimised algorithm also minimises overshoot of the selected flow rate, eliminates flow rate drift, and improves the system response time. In addition to the dramatic improvements in flow rate control, the set up enables the polymer actuation behaviour to be rapidly characterised. The power supply to the LED also provides a useful system diagnostic for monitoring the performance of the valve over time. For example, degradation in the valve actuation due to photodegradation will manifest as an increasing power requirement over time, enabling predictive failure thresholds to be established for particular actuator designs and polymer compositions.
\end{abstract}

Received 4th April 2017,

Accepted 17th May 2017

DOI: $10.1039 / \mathrm{c} 7 \mathrm{lc} 00368 \mathrm{~d}$

rsc.li/loc the human body. ${ }^{6}$ Despite decades of intensive research in chemical sensing, the scale of this challenge is such that there is not a single example of a long-term implantable device available for monitoring and reporting biochemical parameters.

The barrier inhibiting the generalised use of chemical sensors and biosensors for long term monitoring is that they tend to lose calibration rapidly when in continuous contact with complex sample matrices like blood, and regular recalibration is required to correct for baseline drift and changes in sensitivity due to biofilm formation on the sensor surface, and other effects. ${ }^{7,8}$ It seemed to many that microfluidics offered a way to solve this issue by enabling remote calibration of these sensors using small reagent volumes, provided effective ways to control fluid handling could be implemented. Unfortunately this has not happened, and the delivery of functional platforms for long-term monitoring remains largely unrealised. ${ }^{5}$ More detail on the materials challenges associated with implantable devices can be found in a recent review by Scholten and Meng. ${ }^{9}$

In recent years, a number of teams have investigated the use of photoresponsive polymers for providing valve function in microfluidics platforms. This approach is attractive in that the control stimulus (light) requires no physical contact with the actuator, unlike electronic stimuli, which require contact structures to be integrated into fluidic system during fabrication. Initial reports were encouraging, demonstrating that

\footnotetext{
${ }^{a}$ Insight Centre for Data Analytics, National Centre for Sensor Research, Dublin City University, Dublin 9, Ireland. E-mail: dermot.diamond@dcu.ie

${ }^{b}$ School of Mechanical and Design Engineering, Dublin Institute of Technology,

Bolton Street, Dublin, Ireland

$\dagger$ Electronic supplementary information (ESI) available. See DOI: 10.1039/ c7lc00368d
} 
valve behaviour could be implemented using these soft photoresponsive gels. For example, Sumaru et al. showed that a spiropyran-functionalised pNIPAAm gel could be photoactuated in a channel to produce a valve function. ${ }^{10}$ The mechanism involved photoconversion between the non-polar spiropyran and the much more hydrophilic protonated merocyanine isomer. We demonstrated similar ionogel structures that contracted by up to $40 \%$ volume when exposed to light. ${ }^{11}$ In both cases contraction was rapid, occurring in seconds, but recovery (expansion) took much longer, in the order of $10 \mathrm{~s}$ of minutes due to the slow rate of water diffusion into these relatively large structures. Subsequently, much faster actuation rates were achieved by varying the spiropyran derivative and the composition of the gel. ${ }^{12,13}$ Incorporation of acrylic acid to provide a source of protons internal to the gel, led to enhanced switching performance due to improved actuation kinetics, and extension of the effective $\mathrm{pH}$ range to ca. 7.5, as acidified solutions were no longer required to protonate the merocyanine isomer. ${ }^{14}$ The resulting gels could be switched between open/closed repeatedly using light, with good reproducibility. ${ }^{15}$

More recently, we have explored ways to extend this effect towards control of flow rate, rather than switching between fully open/close states. We found that the gels could be poised at intermediate states of expansion by pulsing the LED to provide variable degrees of photostimulation to the gel. ${ }^{16}$ However, these results, while promising, exhibited classic control issues including overshoot of the set point and lack of stability of the steady-state flow rate. In process engineering, the classic way to tackle such issues is to invoke variants of the well-known PID control algorithm. ${ }^{17}$ In this paper, we report on very significant improvements in flow control performance obtained by implementing PID control of the power provided to the LED illuminating the hydrogel actuator (Fig. 1), using real-time flow rate measurements as the control variable.

\section{Experimental}

\section{Materials}

$N$-Isopropylacrylamide $\quad 98 \% \quad$ (NIPAAm), $\quad N, N^{\prime}$-methylenebisacrylamide 99\% (MBIS), phenylbis(2,4,6 trimethyl benzoyl) phosphine oxide 97\% (PBPO), acrylic acid (180-200 ppm MEHQ as inhibitor) 99\% (AA), tetrahydrofuran 99\% (THF), anhydrous dichloromethane (50-150 ppm amylene as stabilizer) 99\% (DCM), were acquired from Sigma Aldrich, Ireland and used as received. The spiropyran derivative 6-((1',3',3'-trimethylspiro[chromene-2,2'-indolin]-6-yl)oxy)hexyl acrylate (SPA) (SP-A) was synthesised as described elsewhere. ${ }^{12}$ Microfluidic chips were fabricated, as described below, from $2 \mathrm{~mm}$ extruded clear poly(methyl methacrylate) (PMMA) sheets supplied by Access Plastics (Ireland). Kingbright $\mathrm{HB}$ Blue $450 \mathrm{~nm}$ LED lights, the Arduino Uno i2c board and all wires were purchased from Farnell components (Ireland). The 12-bit analog to digital converter (ADC) ADS1015 and the pulse wave modulation (PWM) interface
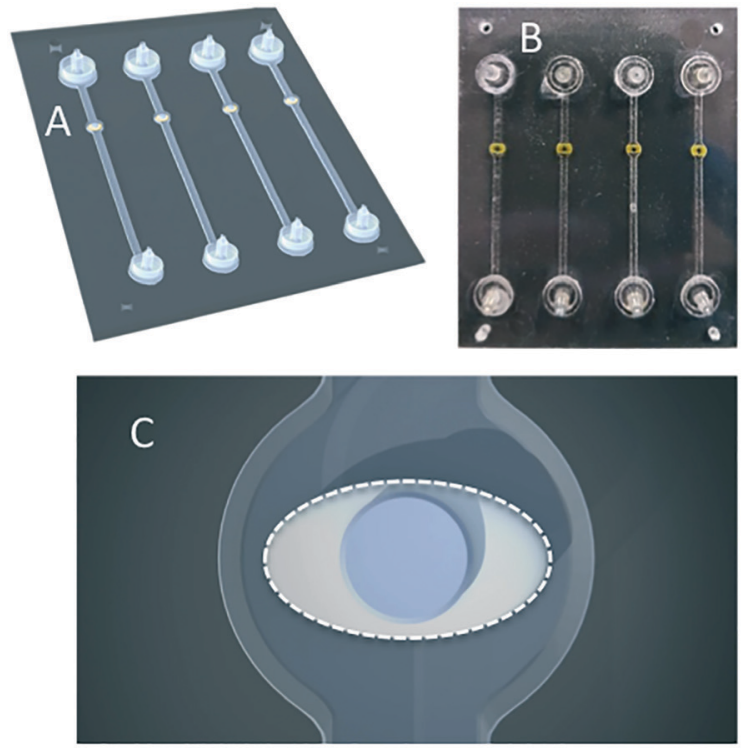
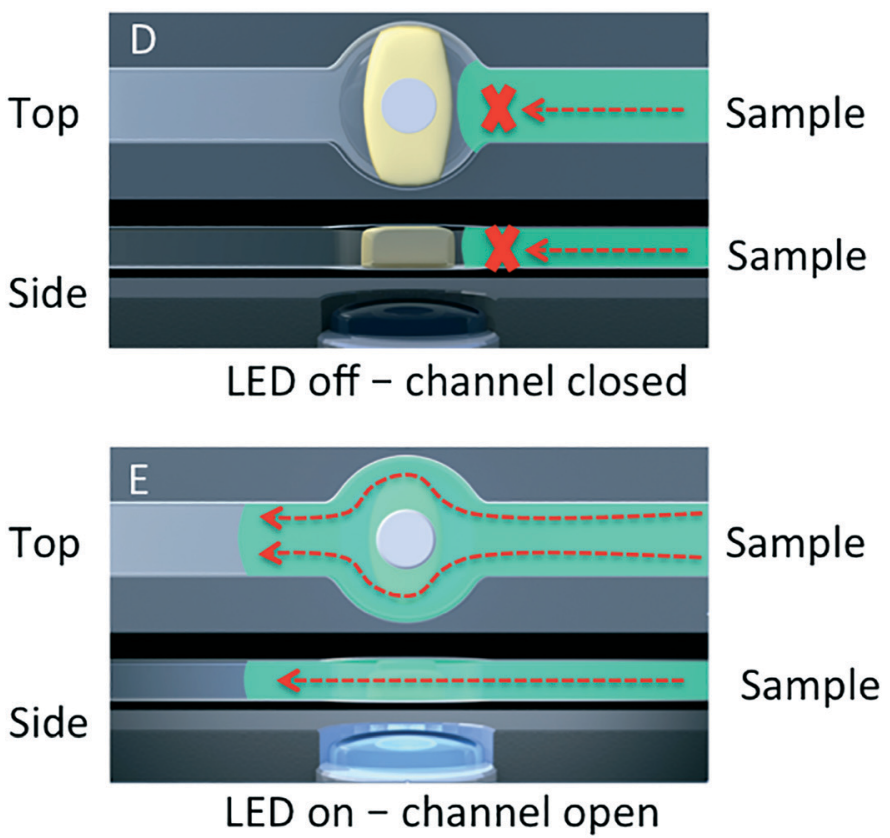

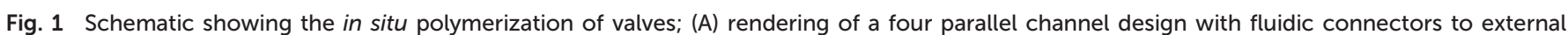

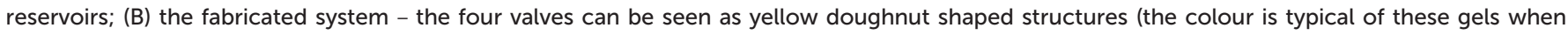

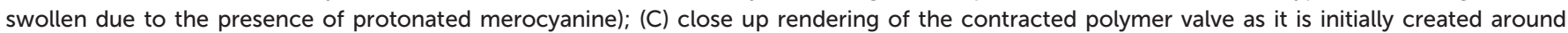

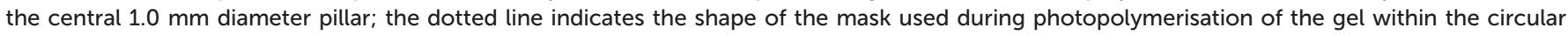

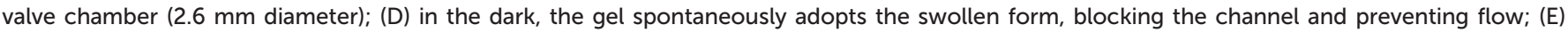
when the LED is turned on, the spiropyran isomer is formed, triggering contraction of the gel and the channel opens. 
PCA9685 were both purchased from Adafruit Technologies. Femtobuck constant-current LED Drivers were purchased from SparkFun Electronics. The printed circuit board (PCB) was supplied by PCB-Pool (http://www.pcb-pool.com/ppuk/). The system ABS box with top plate and fluidic chip alignment pins were obtained from The Laser Company, Clonshaugh Industrial Estate, Dublin 17, Ireland.

\section{Control electronics and software}

The LED control platform (Fig. 2) operated via a 12-bit 16 channel PWM controller (PCA9685) connected to an Arduino Uno microcontroller using the i2c protocol (resolution 0 -4096). An output value of 4096, equivalent to an output current of $150 \mathrm{~mA}$, set the LED at $100 \%$ intensity. Fig. 2 shows a schematic of the four constant current drivers which controlled the LEDs used to actuate four polymer actuators, in which each driver was connected to a different output of the PWM controller, thereby allowing individual control of each photoactuated valve. A UART (serial) connection between the Arduino and the laptop enabled the control software to send the PWM value used to maintain or vary the flow rate. The control software allowed for pre-defined command files to be used through which the required flow rate, proportional constant, integration constant and maximum intensity for a given LED could be set. The system was powered via a $12 \mathrm{~V}$ adaptor and connected to a laptop via a USB port.

A Fluigent flow rate platform (FRP) sensor coupled with a medium flow unit sensor (http:/www.fluigent.com/flow-rate- platform/) was used to track the flow rate in real time. The FRP was connected to a laptop via a USB cable and the data transferred as a binary string. Data was sampled from the FRP at a frequency of $10 \mathrm{~Hz}$ and a boxcar average, with an interval of $1 \mathrm{~Hz}$, was applied. This allowed the control software to record a value every $1 \mathrm{~s}$ while simultaneously improving the signal to noise ratio. Flow control software was subsequently developed in-house, using C-Sharp programming language, which allowed for development of the graphical user interface (GUI) and implementation of the PID control algorithm.

\section{Fluidic system}

The experimental setup used during the experiments is shown in Fig. 3. A constant-head of pressure established between two cylindrical containers positioned on adjustable height stages was used to maintain reproducible, pulseless flow through the microfluidic channels. Fluidic connection to the chip was achieved using Tygon tubing with an internal diameter of 0.8 $\mathrm{mm}$ and connectors located on the chip surface.

The hydrogel valves were photopolymerised in situ from a monomeric cocktail which consisted of $200 \mathrm{mg}$ NIPAAm, 8.35 $\mathrm{mg}$ MBIS (3 mol\% relative to NIPAAm), $7.91 \mathrm{mg}$ SPA-8 (1 mol\% relative to NIPAAm), $7.42 \mathrm{mg}$ PBPO ( $1 \mathrm{~mol} \%$ relative to NIPAAm) and $6.05 \mu \mathrm{L}$ AA ( 5 mol $\%$ relative to NIPAAm) dissolved in $500 \mu \mathrm{L}$ of the polymerisation solvent $(2: 1 \mathrm{v} / \mathrm{v}$, THF : DI water). Details of the fluidic system, valve structures, electronics and photonic arrangements have been described previously, ${ }^{16}$ and are summarised below.
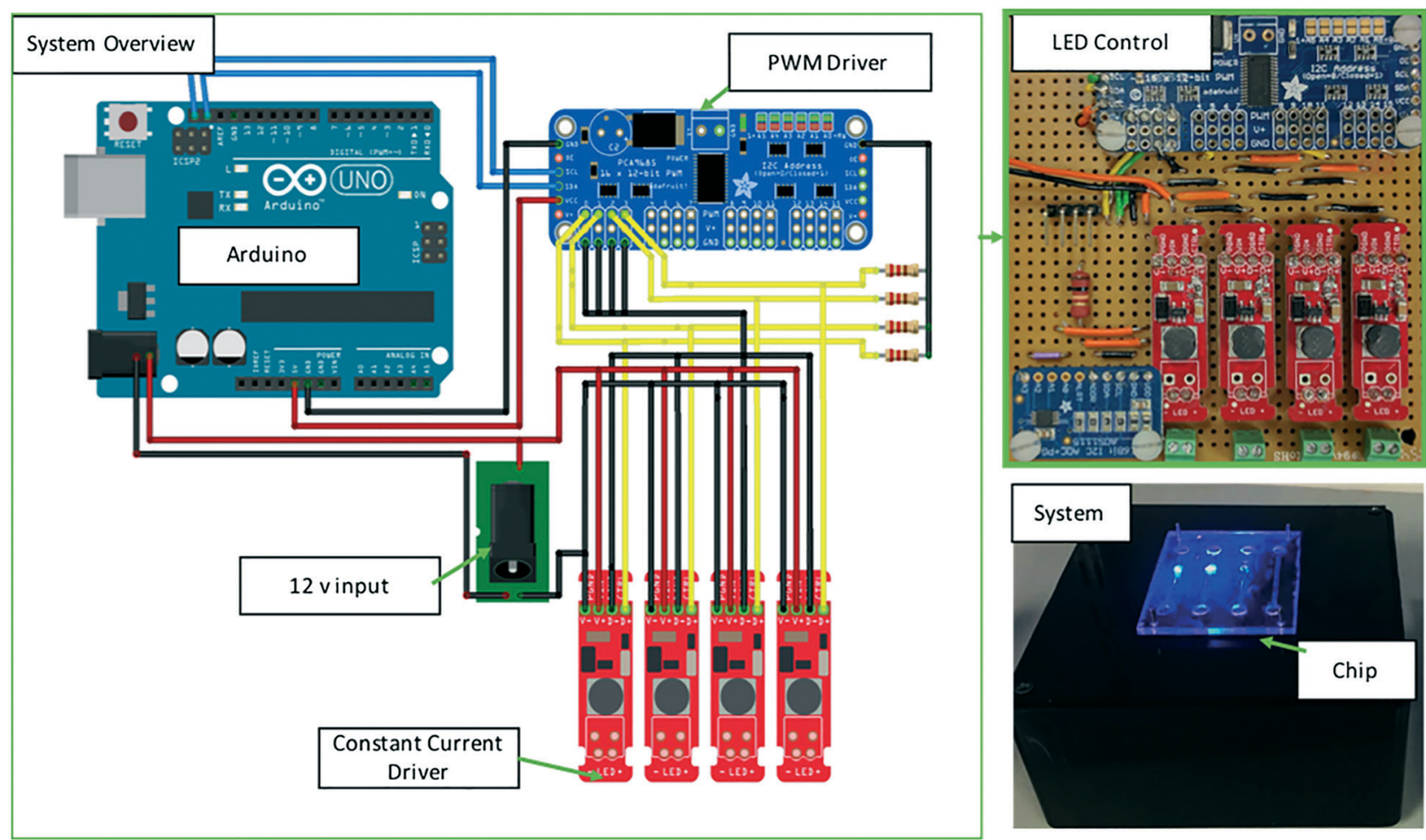

Fig. 2 Design of the actuation module, showing wiring diagram for the Arduino Uno microcontroller (left); physical implementation of the circuitry (right, top) which is placed inside the black PMMA module casing (shown with top open). When closed, the microfluidic chip is located with the valves accurately located above the LEDs (right, bottom). 


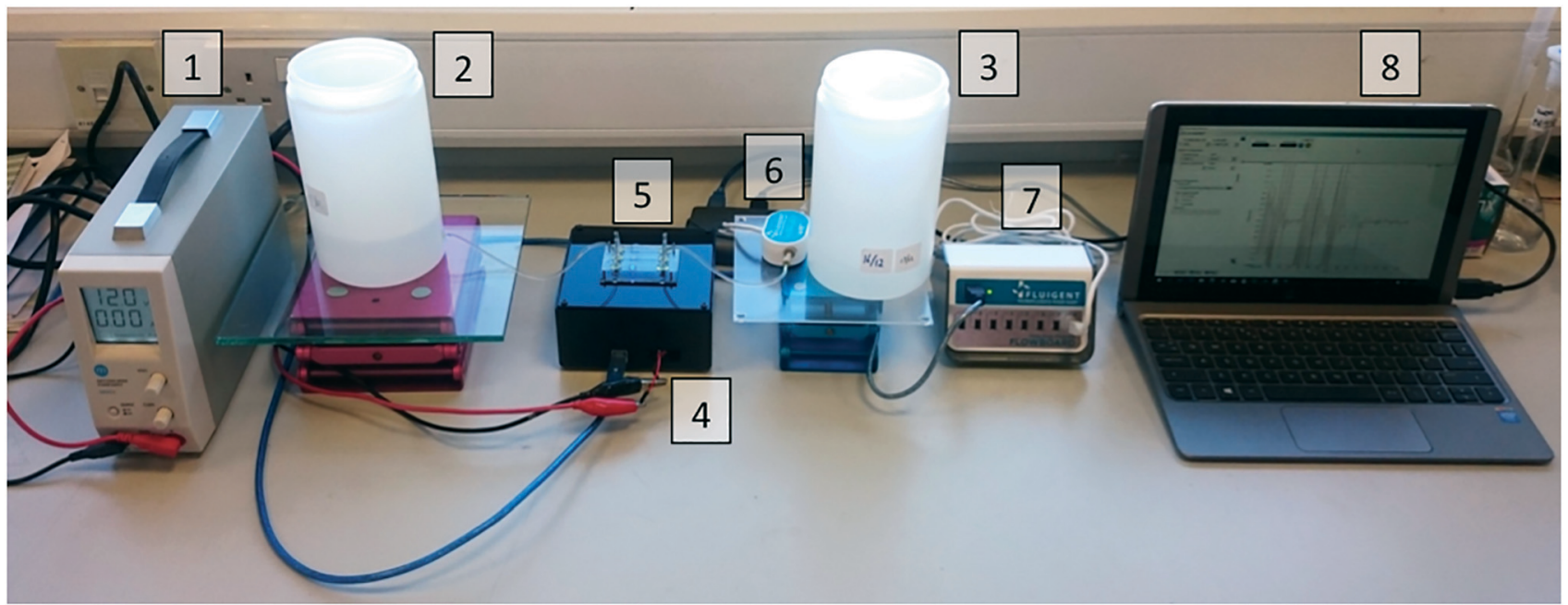

Fig. 3 Experimental set-up for flow-rate experiments showing; 1: power supply; 2 and 3: reagent reservoirs on adjustable height units; 4: inhouse developed electronics control unit; 5: fluidic chip; 6 and 7: Fluigent flow sensor and electronics; 8: laptop.

The microfluidic chips were designed using AutoCAD 2014 and fabricated using clear 2 mm PMMA. Channels were rastered using a $\mathrm{CO}_{2}$ laser ablation system (Epilog Zing Laser Series) at dimensions of $1 \mathrm{~mm}$ wide and $200 \mu \mathrm{m}$ deep. The valve chamber comprised a $1 \mathrm{~mm}$ diameter pillar centred within a circle of diameter $2.6 \mathrm{~mm}$ which extended from the wall of the channel, as seen in Fig. 1. The channels were sealed with a $1 \mathrm{~mm}$ PMMA capping layer using previously described solvent bonding processes. ${ }^{16}$ A series of $1.6 \mathrm{~mm}$ polysulfone Nordson barbs were then attached at both inlet and outlet ports. The monomer cocktail was introduced to the microfluidic channel using a syringe and both ports sealed to inhibit the movement of liquid. Polymerisation was achieved using blue surface-mounted LEDs (Kingbright HB Blue 450 $\mathrm{nm}, 600 \mathrm{~mW} / 4.5 \mathrm{~lm} / 1.3 \mathrm{~cd}, 3.5 \mathrm{~V}$ ) at $450 \mathrm{~nm}$ wavelength and $10 \%$ power for $15 \mathrm{~s}$ through a $1.8 \mathrm{~mm} \times 1.25 \mathrm{~mm}$ elliptical mask, as shown in Fig. 1. Unreacted monomer solution was removed from the channels by flowing deionised water

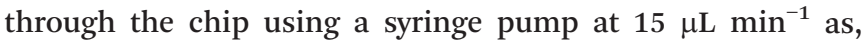
when initially formed, the valves are in the contracted state and do not block the channel. The valves were polymerised around the cylindrical pillar which provided a physical anchor to prevent movement of the valve.

The elliptical valve profile was adopted as more symmetrical circular/cylindrical profiles were found to expand into the channels and become lodged in position, permanently blocking the liquid flow. Once the unreacted monomer solution had been removed, the channels were filled with deionised water and left overnight to ensure full hydration of the hydrogel valves. As outlined previously, ${ }^{16}$ a constant head of pressure was maintained throughout all the experiments using two reservoirs of different volumes, shown in Fig. 3. The source reservoir, which fed to the microfluidic chip, was held at a constant volume of $500 \mathrm{~mL}$ at a height of $62 \mathrm{~mm}$ from the channel inlet. At the outpoint of the channel, a collection reservoir, of $300 \mathrm{~mL}$ volume, was connected at a height of $29 \mathrm{~mm}$ from the output port, providing an overall differential pressure of 3.23 mbar. $^{16}$ Using relatively large reservoirs of this nature ensured that, even over prolonged experiments, only minimal changes occurred in the water levels and therefore the pressure. For example, a flow of $10.0 \mu \mathrm{L} \min ^{-1}$ over a period of 90 minutes produces a transfer of less than $0.9 \mathrm{~mL}$ between the two reservoirs.

\section{Valve actuation}

Actuation of the valves was performed with the same LEDs (Kingbright HB Blue $450 \mathrm{~nm}, 600 \mathrm{~mW} / 4.5 \mathrm{~lm} / 1.3 \mathrm{~cd}, 3.5 \mathrm{~V}$ ) and in-house developed illumination module (Fig. 2) used during the photopolymerisation stage. The four LEDs were located beneath the fluidic chip directly opposite the valves using location stubs, with the illumination intensity controlled via constant current LED drivers and Arduino Uno microcontroller, as described previously. The same masks used to create the hydrogel valves were used to direct the LED light onto the valves. Effective valve control required a high degree of accuracy and reproducibility for the alignment of the LEDs and the valve structures. Accurate placing of the LEDs onto the printed circuit board (PCB) required the use of a reflow soldering method with an optimal reflow temperature of $245{ }^{\circ} \mathrm{C}$. A hot air rework soldering station was used to achieve this via a plume of funnelled hot air emitted from its nozzle. The temperature and air flow rate from the nozzle could be adjusted on the station to provide the required heating pattern for precise location of the LEDs.

\section{Implementing control}

Eqn (1) shows the PID control algorithm applied to flow control of our system; 


$$
P_{\mathrm{LED}}=K_{\mathrm{P}}\left(F_{\mathrm{SP}}-F_{\mathrm{M}}\right)+K_{\mathrm{I}} \int\left(F_{\mathrm{SP}}-F_{\mathrm{M}}\right) \mathrm{d} t+K_{\mathrm{D}}\left(\frac{\mathrm{d}\left(F_{\mathrm{SP}}-F_{\mathrm{M}}\right)}{\mathrm{d} t}\right)
$$

where $P_{\mathrm{LED}}=$ LED power input, $K_{\mathrm{P}}=$ proportional gain $(\mathrm{P})$, $K_{\mathrm{I}}=$ integral gain $(\mathrm{I}), K_{\mathrm{D}}=$ derivative gain $(\mathrm{D})$ and $\left(F_{\mathrm{SP}}-F_{\mathrm{M}}\right)=$ error between the flow rate set point $\left(F_{\mathrm{SP}}\right)$ and the measured flow rate $\left(F_{\mathrm{M}}\right)$. The derivative element of the control algorithm was not implemented in this study; i.e. PI control was used rather than full PID control, with $K_{\mathrm{D}}$ set to zero throughout.

To implement real-time control, the variable to be controlled (flow rate) must be measured in real-time. In our experimental setup, access to flow rate data was provided by the Fluigent sensor and electronics (6 and 7; Fig. 3). This was passed to the laptop, where in-house developed software calculated the appropriate power setting for the LED as a pulse wave modulation (PWM) value which was sent to the PWM driver via the Arduino board (Fig. 2). The PWM value sets the power provided to the LED, and this was varied according to the error between the set point (desired flow rate) and the variable value (measured flow rate) according to the PI algorithm.

The sensitivity of the algorithm elements is defined by the proportional $\left(K_{\mathrm{P}}\right)$ and integral gain $\left(K_{\mathrm{I}}\right)$ values. Optimisation involves varying these constants to maximise the accuracy, precision and response dynamics of the system across a range of target variable values. In this study, this was performed manually using the graphical user interface, (see Fig. S1, $\dagger$ and accompanying text).

Once a reasonably well-optimised set of conditions was achieved using the software, it was possible to load flow sequence files to enable autonomous flow control over extended periods of time.

\section{Results and discussion}

Our motivation to explore the impact of PID control emerged from a previous study, ${ }^{16}$ in which we used a pulsed approach to vary the power provided to an LED to create intermediate states between open and closed for photoactuated valves in a fluidic channel. The results were encouraging, as they clearly demonstrated that it was possible to exert some degree of control on the flow rate. For example, Fig. S2 $\uparrow$ shows three replicate attempts to control the flow rate by pulsing the LED. The behaviour appeared to be surprisingly reproducible, but rapid, precise and accurate control was difficult to achieve. In particular, initial large overshoots of the steady state flow occurred (Fig. S2A $\dagger$ ) resulting in long settlement times, along with slow baseline adjustments towards a longer-term steady state value (Fig. S2B $\dagger$ ). In addition, with this approach it was not possible to predict a priori the steady-state flow rate that would be associated with a particular LED exposure of the valve structure. Instead, an empirical approach had to be employed, without knowledge of how reproducible the flow rate would be for a particular photo-expo- sure, or how stable this flow rate would be during repeated use. This made characterisation of the system response, and associated optimisation of the performance very difficult from a practical perspective.

The pNIPAAm hydrogels used to create these valves function on the basis of water uptake (swelling) and release (contraction) due to LCST switching which is triggered by photoisomerisation of the covalently incorporated spiropyranmerocyanine system. ${ }^{12}$ Previous studies showed that this behaviour could be used to open/close channels in microfluidic systems by using light to cause the hydrogels to contract and swell. However, precise control of an intermediate state to allow flow control is more challenging, as water uptake/release by these hydrogels is unlikely to be a highly reproducible effect, and the flow rate obtained will depend on multiple factors such as the gel composition, variation in the polymer structure/composition during formation and use, the liquid/ sample composition, and the light flux impacting on the polymer. Given these constraints, to make any progress one would have to create very reproducible structures, and these would have to exhibit very reproducible photoactuation characteristics between structures and over time for effective implementation. This would require detailed modelling of numbers of valves under numerous conditions to enable the flow behaviour to be mapped, and the constraints managed.

In contrast, applying PID control based on real-time flow rate data enables the relationship between LED power and flow rate to be rapidly characterised. In process control, overshoots are dealt with by applying proportional control, as this begins to take effect at a point prior to reaching the set point, slowing down the change in the control variable (flow rate) as it approaches the set point. The sensitivity of this effect can be tuned by varying the value of the proportional constant $\left(K_{\mathrm{P}}\right)$; too low a value has little impact and overshoots continue to happen; too high and the effect kicks in too early, leading to long response times and steady-state values that are consistently offset from the desired set point, as the control input is essentially completely off near the set point. The integral factor is designed to detect and compensate for a consistent difference between the set point and the steadystate value, by integrating the difference over a set time, and applying a correction to the input factor (LED power in this case). As is often the case, we obtained excellent results using proportional and integral (PI), control, and the derivative factor was not used in the subsequent experiments.

After preliminary optimisation experiments, it was found that for this particular experimental set up, good results over a range of typical flow rates (up to $15 \mu \mathrm{L} \mathrm{min}{ }^{-1}$ in this research) could be obtained using the values $K_{\mathrm{P}}=10.0$, and $K_{\mathrm{I}}$ $=0.1$, and these values were used during most of the subsequent investigations. Fig. 4 (top) shows the results obtained for 10 replicate switches between zero and $10 \mu \mathrm{L} \mathrm{min}^{-1}$ (see Table S1 $\uparrow$ for summary statistics). A small overshoot occurs in each case which quickly settles to a relatively constant flow rate. The large feature at the first peak is a bubble artefact which can be discounted. Remarkably, the average flow rate 

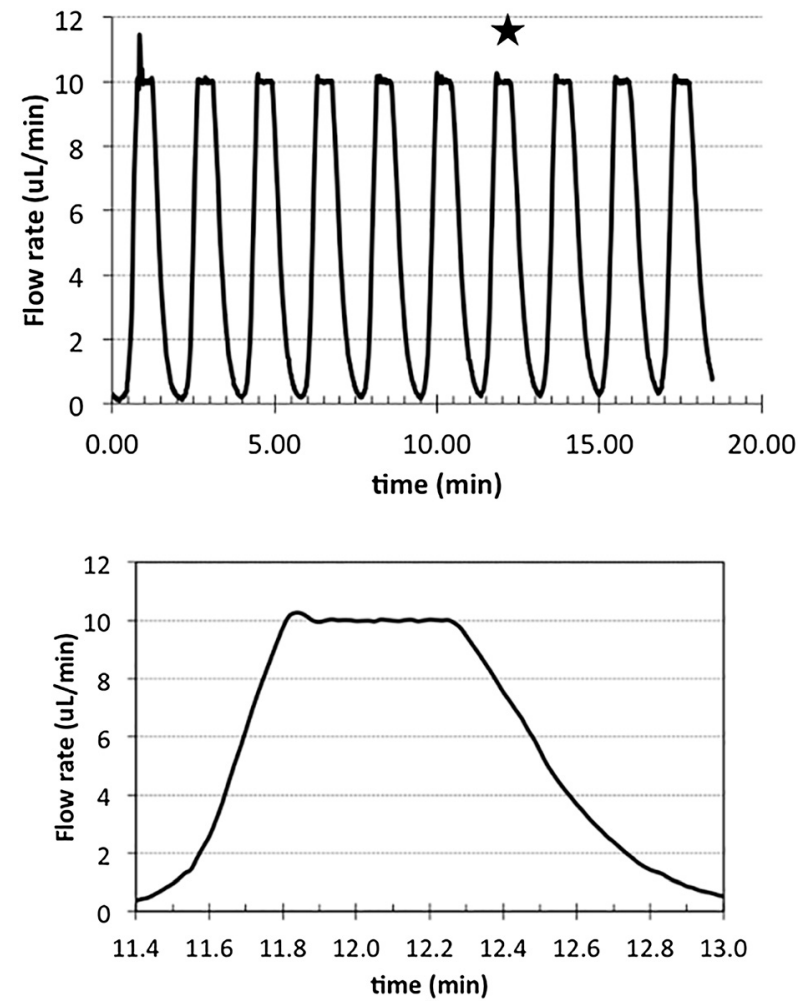

Fig. 4 (Top) - Ten replicate switches between zero and $10.0 \mu \mathrm{L} \mathrm{min}{ }^{-1}$; $K_{\mathrm{P}}=11 ; K_{1}=0.045$; integration period $=10 \mathrm{~s}$. The sharp feature at the leading edge of the first peak is a bubble artefact. See Table $\mathrm{S} 1 \uparrow$ for summary statistics. (Bottom) - Zoom in on peak 7 (starred) showing the rapid response (ca. $30 \mathrm{~s}$ on rise, ca. $50 \mathrm{~s}$ on fall), small overshoot at the leading edge, very stable steady-state flow rate.

(calculated from 15 points taken just after settlement of the small overshoot) is within a few $\mathrm{nL} \mathrm{min}^{-1}$ of the set flow rate, with the average error over the 10 peaks being $2.8 \mathrm{~nL} \mathrm{~min}^{-1}$. The average error modulus is $6.4 \mathrm{~nL} \mathrm{~min}^{-1}$ and the overall average standard deviation is $\pm 32 \mathrm{~nL} \mathrm{m^{-1 }}$. Fig. 4 (bottom) presents a magnified version of peak 7 (starred), in which the greatly reduced overshoot, very stable and accurate steadystate flow-rate, and relatively rapid response ( $c a .30 \mathrm{~s}$ rise, 50 $\mathrm{s}$ fall) is clearly evident.

Fig. 5 shows typical results obtained for switching between set flow rates in the sequence 5.0, 10.0, 15.0, 10.0, 5.0 $\mu \mathrm{L}$ $\mathrm{min}^{-1}$, along with the corresponding LED power data. The effect of small changes to the control algorithm constants is immediately apparent, through the complete removal of the overshoot feature and the relatively rapid establishment of a stable steady-state flow rate for each step, in both directions (increasing and decreasing flow rates). Also shown is the power supply to the LED, which mirrors the flow rate pattern over the period of the experiment (2800 s, $c a .46$ minutes). Table S2 $\uparrow$ presents summary statistics for the experiment. In every case, the steady-state flow rate was slightly above the set flow rate, most likely due to the slight decrease in the $K_{\mathrm{P}}$ value compared to Fig. 4 . This can be compensated for by introducing a correction factor which adjusts the control algorithm parameters so that the steady-state value more closely

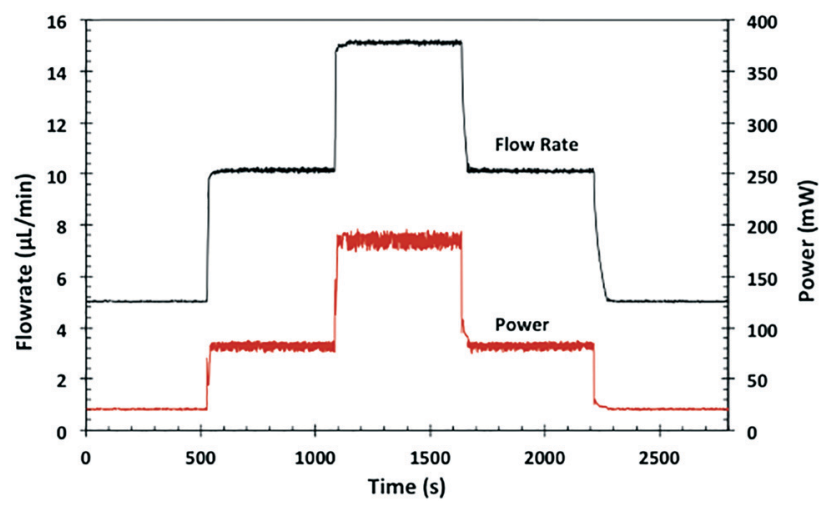

Fig. 5 Flow rate control obtained using $K_{\mathrm{P}}=10.0 ; K_{1}=0.1$. Excellent stability at set flow rates of $5.0 \mu \mathrm{L} \mathrm{min}{ }^{-1}, 10.0 \mu \mathrm{L} \mathrm{min}{ }^{-1}, 15.0 \mu \mathrm{L} \mathrm{min}$, $10.0 \mu \mathrm{L} \mathrm{min}{ }^{-1}$, and $5.0 \mu \mathrm{L} \mathrm{min}{ }^{-1}$ is clearly evident. The overshoot has been completely eliminated. The LED power data mirrors the flow rate with no indication of increasing demand over time.

aligns with the set point. However, across the entire experiment, the offset is relatively small for each step in the flow profile, averaging at $+91 \mathrm{~nL} \mathrm{~min}^{-1}$, and the initial overshoot has been completely eliminated. The flow rate standard deviation is lower $\left(5.0 \mu \mathrm{L} \min ^{-1}\right)$ than at the higher flow rates $\left(<20 \mathrm{~nL} \min ^{-1}\right.$ compared to $\left.c a .50 \mathrm{~nL} \mathrm{~min}{ }^{-1}\right)$, which can be seen as slightly increased baseline noise in Fig. 5. Similarly, the LED power fluctuation also increases, as the algorithm responds dynamically to variations in the flow rate. The LED power shows no evidence of increasing demand over time, and is in fact remarkably constant for a particular flow rate (Table S2†).

Fig. 6 zooms in on the step changes shown in Fig. 5 to enable the dynamics of the changes to be examined more closely. As is common with these gel actuators, expansion is slower than contraction due to hydration effects. ${ }^{15}$ In addition, with this particular experimental design, only the contraction process (conversion of the protonated merocyanine to the uncharged spiropyran) is photo-driven, as the reverse process occurs spontaneously in the absence of light. Nevertheless, for gel actuators of these dimensions, the response dynamics are reassuringly rapid at $c a .10 \mathrm{~s}$ for increasing flow rates, and $c a$. 30-40 s for decreasing flow rates. It is likely that these responses could be further improved by varying the gel formulation and the spiropyran derivative, the illumination strategy (e.g. introducing micro-lens structures to more effectively couple light into the gel), and reducing the dimensions of the flow channels and actuators. ${ }^{12,14,18}$

Fig. 7 shows similar step changes in flow rate repeated over a longer time scale to explore the system stability and reproducibility ( $c a .2 .5$ hours). The results are similar to Fig. 5, indicating that the system is very stable, even in protracted use. Interestingly, the average LED power required to obtain each steady-state flow rate is once again remarkably constant. For example, the mean of the average power values for the $10.0 \mu \mathrm{L} \mathrm{min}{ }^{-1}$ regions in Table $\mathrm{S} 3 \dagger$ is $82.33 \pm 0.15 \mathrm{~mW}(n=$ 8 ), which is a very low \% RSD of $0.18 \%$. The implications of these results are significant. For example, the reproducibility 

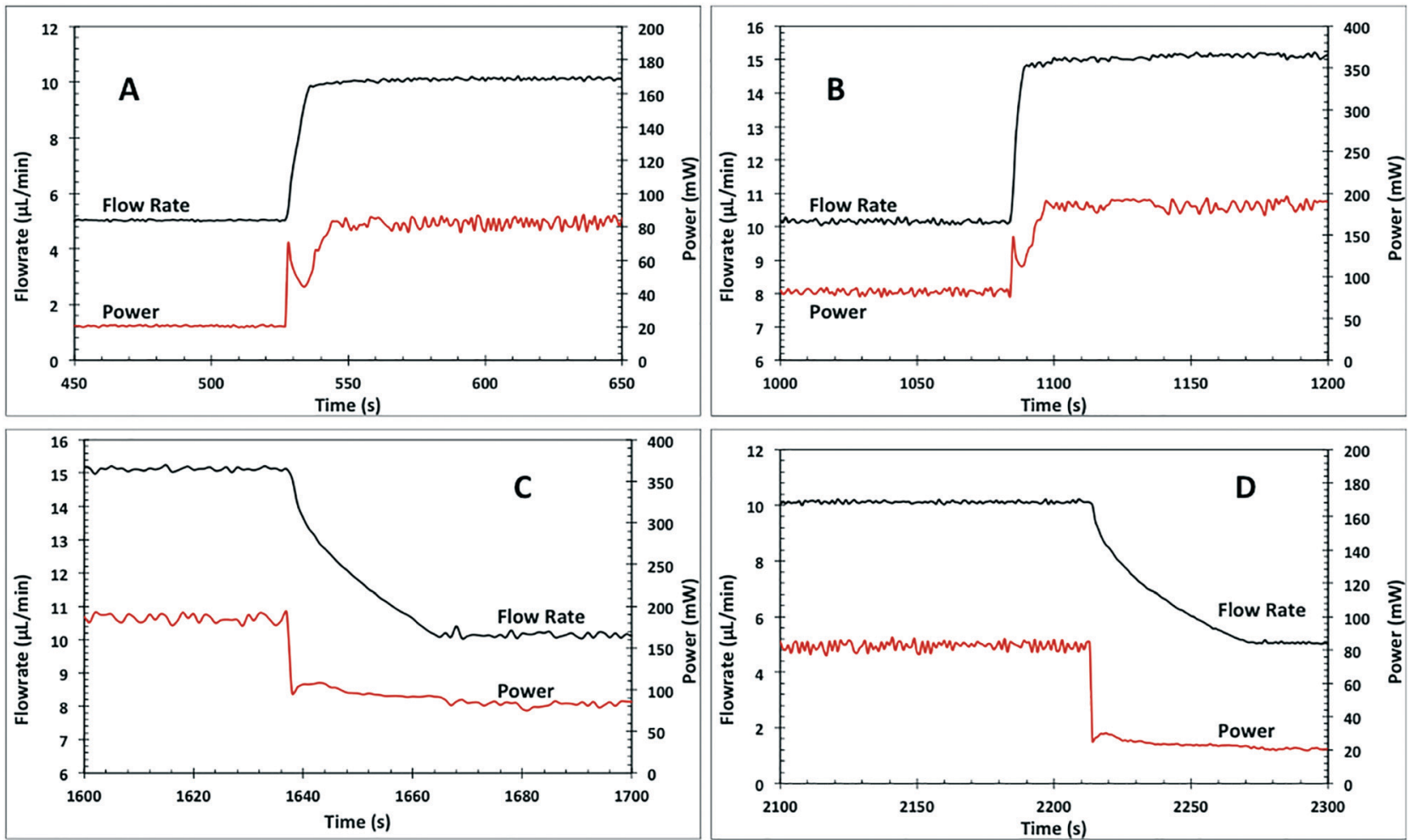

Fig. 6 Dynamic response of flow rate and LED power supply for the step features in Fig. 5. In all cases, the step changes are very rapid, with the increasing steps (A: $5.0-10.0 \mu \mathrm{L} \mathrm{min}{ }^{-1}, \mathrm{~B}: 10.0-15.0 \mu \mathrm{L} \mathrm{min}^{-1}$ ) essentially complete within $10 \mathrm{~s}$, and the decreasing steps (C: 15.0-10.0 $\mu \mathrm{L}$ min ${ }^{-1}$, D:

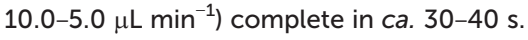

of the LED power for a particular flow rate suggests that it may be possible to predict the flow rate associated with a particular LED power, and therefore direct measurement of flow rate may not be required.

However, given the non-linearity of the relationship between flow rate and LED power evident from these data, and the diagnostic value of the power data, a much more reliable outcome is obtained when the flow rate data is available. But in turn this raises the need for ways to generate accurate flow

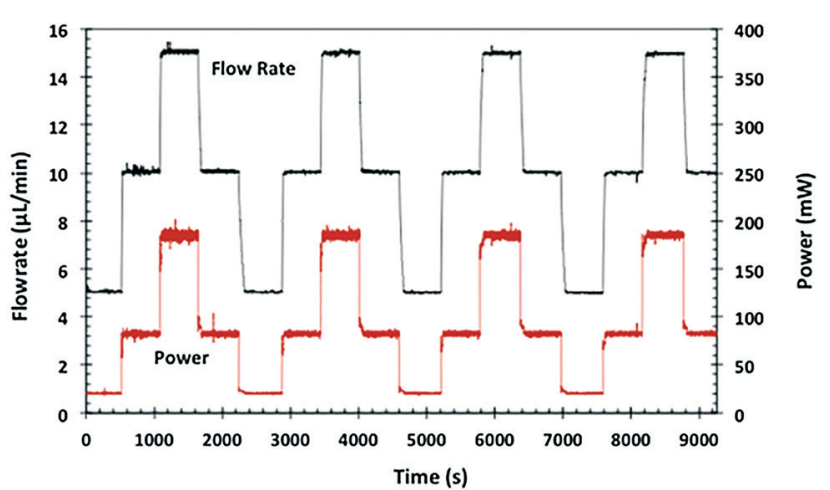

Fig. 7 Four cycles of step changes in flow rate $(5.0,10.0,15.0,10.0$, $5.0 \mu \mathrm{L} \mathrm{min}{ }^{-1}$ ) with the associated changes in LED power over a period of ca. 2.5 hours. $K_{\mathrm{P}}=10.0 ; K_{\mathrm{l}}=0.1$; see Table $\mathrm{S} 3 \uparrow$ for summary statistics. rate data in multiple channels, if the approach is to be scaled up for use in more complex flow systems.

Fig. 8 presents the performance of the system while maintaining a constant flow rate $\left(10.0 \mu \mathrm{L} \mathrm{min}{ }^{-1}\right)$ for $90 \mathrm{mi}-$ nutes. Once again, the results are very impressive, the average flow rate being $10.047 \pm 0.032 \mu \mathrm{L} \mathrm{min}^{-1}(n=1000)$. The precision is excellent $\left( \pm 32 \mathrm{~nL} \mathrm{~min}{ }^{-1}\right)$ and the slightly positive

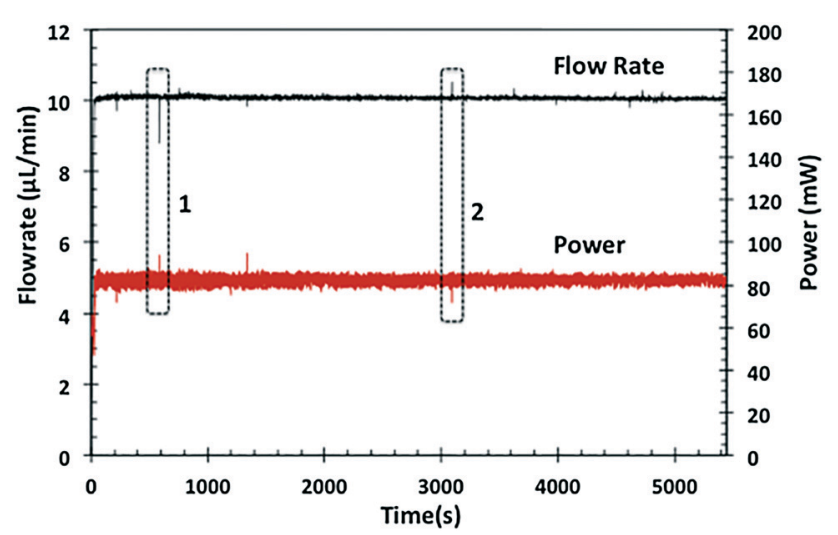

Fig. 8 Steady state flow rate $\left(10 \mu \mathrm{L} \mathrm{min}{ }^{-1}\right)$ and power $(\mathrm{mW})$ provided to the LED for a period of $c a$. 90 minutes. The rapid response of the control algorithm is demonstrated by the features ( 1 and 2 ) highlighted which show a small change in the flow rate, which is accompanied by an immediate response in the LED power, and rapid re-establishment of the set flow rate. $K_{\mathrm{P}}=10.0 ; K_{1}=0.1$. 
average error of $47 \mathrm{~nL} \mathrm{~min}^{-1}$ could likely be reduced by further optimisation of the control parameters and experimental design. Furthermore, the average flow rate and LED power remain remarkably constant throughout the duration of the experiment, indicating that the gel actuator is stable, displaying no evidence of degradation in performance despite constant illumination for the gel throughout the experiment.

These results raise new challenges for researchers interested in fabricating and characterising complex microfluidic systems with multiple integrated valves, such as how the topography of channels and flow sensors should be arranged and optimised. For example, it is possible that a sub-set of flow sensors could be used to characterise a larger population of valves by switching individual or groups of valves and observing the impact on the output flow-patterns obtained from the valves, and applying machine learning approaches to identify the optimum channel/valve/flow sensor arrangement. In addition, for truly autonomous fluidic devices, the issue of how to integrate flow generation must also be considered. In keeping with the overall focus on biological models, it would be very interesting to integrate fluid movement based on bio and/or bio-like actuators, such as beating heart tissue, ${ }^{19}$ flexible artificial cilia, ${ }^{20}$ or chemically powered micropumps ${ }^{21,22}$ with soft polymer valves to create a fully functional biomimetic fluidic system.

\section{Conclusions}

Using real-time flow rate measurements with PID control to regulate the illumination of photo-responsive gel actuators enables photo-control of flow in microfluidic channels of unprecedented accuracy, precision and stability. This is a significant outcome as it enables flow behaviour in fluidic systems to be controlled externally using light, without the need for any physical connection between the flow regulation components and the control stimulus. The photo-responsive gel actuators can be fabricated very simply in pre-selected locations using masks and LEDs by passing the monomer cocktail through the previously produced fluidic system. This approach is amenable to low-cost scale-up in terms of the numbers of actuators and their precise locations within a fluidic system.

Tracking of the correlation between LED power and flow rate over time provides a powerful system diagnostic that can be used to detect deterioration in actuator performance, and flag issues before serious malfunctions occur. Initial data suggest the actuator stability is excellent, with no indication of deterioration over a period of several hours continuous illumination. Switching between flow rates in the range 0-15

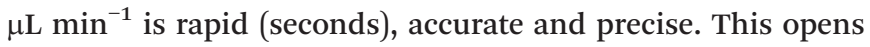
the way to using light to control flow behaviour in more complex fluidic arrangements. For example, coupling flexible display technologies with flexible fluidic layers ${ }^{23}$ could enable the creation of platforms with sophisticated fluidic functions (sampling, reagent addition, distributed measurements \& therapeutic release) that are relevant to applications in personalised health monitoring using on-skin/on-body measurements. As ICT companies increasingly seek new sources of information and health related industries search for more cost-effective treatment options for people with chronic conditions, ${ }^{24}$ these types of platforms will open new possibilities that appeal to both ICT and the healthcare industries. ${ }^{25}$

The scalability of the approach depends on access to distributed flow rate information across the fluidic system. Therefore, achieving ways to perform accurate and scalable flow rate measurements at low cost is an important requirement. For example, the use of distributed colour measurement may be a possibility, ${ }^{26}$ or low cost versions of temperature differential sensors of the type used in this study. In these preliminary studies, the results suggest that, for a well characterised system, it may be possible to predict the flow rate that will be generated for a particular LED power with reasonably good accuracy. While this would remove the need for localised measurement of flow rate, it does mean the user will in a sense be 'flying blind', trusting the stability of the flow regulation over time for a particular application. The feasibility of these ideas will only become clear as more research into the approach happens, and more knowledge is generated about the impact of fabrication strategy, actuator design and dimensions, sample composition, gel formulation and stability and coupling of photonics/fluidics.

In conclusion, we believe this is a very exciting development that will have a significant influence on future research and applications of microfluidics.

\section{Acknowledgements}

Funding for this research has been provided under the European Union Seventh Framework Programme (FP7) through the NAPES project (grant agreement no. 604241), and through the Science Foundation Ireland INSIGHT Centre for Data Analytics (Grant Number SFI/12/RC/2289). We acknowledge assistance and support from Fluigent with accessing flow sensor data, and Mats Bjorklund at http://magipics.com.au/ for original graphics used in Fig. 1.

\section{Notes and references}

1 I. E. Araci and S. R. Quake, Lab Chip, 2012, 12, 2803.

2 A. R. Wheeler, Science, 2008, 322, 539-540.

3 C. K. W. Koo, F. He and S. R. Nugen, Analyst, 2013, 138, 4998.

4 D. T. Eddington, R. H. Liu, J. S. Moore and D. J. Beebe, Lab Chip, 2001, 1, 96.

5 E. K. Sackmann, A. L. Fulton and D. J. Beebe, Nature, 2014, 507, 181-189.

6 S. Coleman, L. Florea and D. Diamond, Inst. Chem. Irel., J., 2016, 13-23.

7 A. A. Abraham, R. Fei, G. L. Coté and M. A. Grunlan, ACS Appl. Mater. Interfaces, 2013, 5, 12832-12838.

8 R. Fei, A. K. Means, A. A. Abraham, A. K. Locke, G. L. Coté and M. A. Grunlan, Macromol. Mater. Eng., 2016, 301, 935-943. 
9 K. Scholten and E. Meng, Lab Chip, 2015, 15, 4256-4272.

10 S. Sugiura, K. Sumaru, K. Ohi, K. Hiroki, T. Takagi and T. Kanamori, Sens. Actuators, A, 2007, 140, 176-184.

11 F. Benito-Lopez, R. Byrne, A. M. Răduță, N. E. Vrana, G. McGuinness and D. Diamond, Lab Chip, 2010, 10, 195-201.

12 J. ter Schiphorst, S. Coleman, J. E. Stumpel, A. Ben Azouz, D. Diamond and A. P. H. J. Schenning, Chem. Mater., 2015, 27, 5925-5931.

13 T. Satoh, K. Sumaru, T. Takagi and T. Kanamori, Soft Matter, 2011, 7, 8030.

14 B. Ziolkowski, L. Florea, J. Theobald, F. Benito-Lopez and D. Diamond, Soft Matter, 2013, 9, 8754-8760.

15 B. Ziolkowski, L. Florea, J. Theobald, F. Benito-Lopez and D. Diamond, J. Mater. Sci., 2016, 51, 1392-1399.

16 S. Coleman, J. ter Schiphorst, A. Ben Azouz, S. Bakker, A. P. H. J. Schenning and D. Diamond, Sens. Actuators, B, 2017, 245, 81-86.

17 K. J. Åström and T. Hägglund, Advanced PID Control, ISA-The Instrumentation, Systems, and Automation Society, 2006.

18 L. Ionov, Mater. Today, 2014, 17, 494-503.
19 Y. S. Zhang, J. Aleman, A. Arneri, S. Bersini, F. Piraino, S. R. Shin, M. R. Dokmeci and A. Khademhosseini, Biomed. Mater., 2015, 10, 34006.

20 Y. Wang, J. den Toonder, R. Cardinaels and P. Anderson, Lab Chip, 2016, 16, 2277-2286.

21 C. Zhou, H. Zhang, Z. Li and W. Wang, Lab Chip, 2016, 16, 1797-1811.

22 D. Patra, S. Sengupta, W. Duan, H. Zhang, R. Pavlick and A. Sen, Nanoscale, 2013, 5, 1273-1283.

23 L. Florea, C. Fay, E. Lahiff, T. Phelan, N. E. O'Connor, B. Corcoran, D. Diamond and F. Benito-Lopez, Lab Chip, 2013, 13, 1079.

24 Adam Brown and Kelly Close, Abbott's FreeStyle Libre Transforming Glucose Monitoring Through Utter Simplicity, Fingersticks Aside!, http://diatribe.org/abbott-freestyle-libretransforming-glucose-monitoring-through-utter-simplicityfingersticks, (accessed March 20, 2017).

25 N. M. Farandos, A. K. Yetisen, M. J. Monteiro, C. R. Lowe and S. H. Yun, Adv. Healthcare Mater., 2015, 4, 792-810.

26 G. Matzeu, C. Fay, A. Vaillant, S. Coyle and D. Diamond, IEEE Trans. Biomed. Eng., 2016, 63, 1672-1680. 